\title{
Interaction of Borrelia burgdorferi with Peripheral Blood Fibrocytes, Antigen-Presenting Cells with the Potential for Connective Tissue Targeting
}

\author{
Dennis J. Grab, ${ }^{1,2}$ H.-Norbert Lanners, ${ }^{1}$ Louis N. Martin, ${ }^{1}$ \\ Jason Chesney, ${ }^{3}$ Chunbo Cai, ${ }^{3}$ Huston D. Adkisson, ${ }^{4}$ and \\ Richard Bucala ${ }^{3}$ \\ ${ }^{1}$ Tulane Regional Primate Research Center, Covington, Louisiana, U.S.A. \\ ${ }^{2}$ Tulane University School of Public Health and Tropical Medicine, New \\ Orleans, Louisiana, U.S.A. \\ ${ }^{3}$ The Picower Institute for Medical Research, Manhasset, New York, U.S.A. \\ ${ }^{4}$ Renal Division, Barnes-Jewish Hospital, Washington University \\ Medical School, St. Louis, Missouri, U.S.A. \\ Communicated by R. Bucala. Accepted December 8, 1998.
}

\begin{abstract}
Background: Borrelia Burgdorferi has a predilection for collagenous tissue and can interact with fibronectin and cellular collagens. While the molecular mechanisms of how B. burgdorferi targets connective tissues and causes arthritis are not understood, the spirochetes can bind to a number of different cell types, including fibroblasts. A novel circulating fibroblast-like cell called the peripheral blood fibrocyte has recently been described. Fibrocytes express collagen types I and III as well as fibronectin. Besides playing a role in wound healing, fibrocytes have the potential to target to connective tissue and the functional capacity to recruit, activate, and present antigen to $\mathrm{CD}^{+} \mathrm{T}$ cells.

Materials and Methods: Rhesus monkey fibrocytes were isolated and characterized by flow cytometry. $B$. burgdorferi were incubated with human or monkey fi-
\end{abstract}

brocyte cultures in vitro and the cellular interactions analyzed by light and electron microscopy. The two strains of $B$. burgdorferi studied included JDl, which is highly pathogenic for monkeys, and M297, which lacks the cell surface OspA and OspB proteins.

Results: In this study, we demonstrate that B. burgdorferi binds to both human and monkey (rhesus) fibrocytes in vitro. This process does not require OspA or OspB. In addition, the spirochetes are not phagocytosed but are taken into deep recesses of the cell membrane, a process that may protect them from the immune system.

Conclusions: This interaction between $B$. burgdorferi and peripheral blood fibrocytes provides a potential explanation for the targeting of spirochetes to joint connective tissue and may contribute to the inflammatory process in Lyme arthritis.

\section{Introduction}

Lyme disease is a tick-transmitted multisystemic disorder in humans (and other mammals) caused by the spirochete Borrelia burgdorferi (1). The bacteria are transmitted to humans by the bite of

Address correspondence and reprint requests to: Dr. Dennis J. Grab, Department of Applied Biological Sciences, Saga University, Saga 840-8502, Japan. Phone: 81-952-28-8775; Fax: 81-952-28-8709; E-mail: grab@cc.saga-u.ac.jp infected ticks of the Ixodes ricinus complex. In North America, Lyme disease is the most frequently reported arthropod-borne infection accounting for over $80 \%$ of all vector-borne infections in the United States (2). If left untreated, one major long-term manifestation of Lyme disease is chronic arthritis. Little is known about the mechanisms used by B. burgdorferi to target connective or other tissues. Although some spiro- 
chetes inevitably reach tissues passively via the circulatory system, other perhaps more specific targeting mechanisms may be involved.

$B$. burgdorferi bind or produce proteolytic enzymes, and several reports describe the ability of B. burgdorferi to bind to host-derived plasminogen. Although the bacteria do not directly activate bound plasminogen, bound plasminogen can be converted to a potent serine protease, plasmin, by host-derived plasminogen activators (3-7). B. burgdorferi possess endogenous collagenase(s) (8) and proteoglycanase (9) activities and there is also evidence to suggest that the spirochetes possess hemolytic activity (10). These enzymatic activities are capable of degrading the extracellular matrices of cells and tissues and they may play an important role in the process of bacterial dissemination and tissue invasion. Nevertheless, the tropism of spirochetes for joints remains poorly understood.

Borrelia burgdorferi interacts with a variety of cells, including fibroblasts (11-13). For example, it has been postulated that the binding of $B$. burgdorferi to activated platelets might favor the concentration of spirochetes to regions of endothelial damage, thereby targeting the spirochetes to the arthropod vector at the site of the tick bite and tick attachment (14). A novel leukocyte subpopulation with fibroblast-like properties called "fibrocytes" has been described recently (15). Both human and mouse fibrocytes are negative for many B and T lymphocyte, macrophage, and dendritic cell markers (i.e., CD3, CD4, CD8, CD16, CD 19, CD25, CD33, CD38, CD44) but are positive for the hematopoetic progenitor cell marker CD34 (15). In addition, fibrocytes express the common leukocyte marker CD45 as well as the fibroblast products collagen type I, collagen type III, vimentin, and fibronectin (15). Fibrocytes are present in connective tissue scars and have the ability to rapidly enter from blood into subcutaneously implanted wound chambers (15). Because of the known interaction of $B$. burgdorferi with fibroblasts (11-13) and fibronectin $(16,17)$, we predicted that the spirochete would also interact with fibrocytes. The predilection of both $B$. burgdorferi $(13,18,19)$ and fibrocytes (15) for connective tissues led us to speculate that the spirochete may utilize the fibrocyte as a means to reach joints from the peripheral circulation. We provide here morphologic evidence for spirochete/fibrocyte interactions, an important prerequisite for fibrocyte-mediated spirochete targeting.

\section{Materials and Methods}

Materials

Rabbit anti-human collagen immunoglobulin G (IgG) (No. T61554R: a mixture of anti-human type I, II, III, IV, and V IgGs) was purchased from Biodesign (Kennebunk, ME). Fluorescein (FITC)conjugated sheep anti-rabbit IgGAM (No. PF310) was obtained from The Binding Site (San Diego, CA), Phycoerythrin-conjugated anti-CD34 monoclonal antibody (MAb) was from Becton Dickinson (Bedford, MA), and FITC-conjugated anticollagen type I MAb was from Chemicon (Temecula, CA). Rabbit serum was obtained from Pel-Freeze Biologicals (Rogers, AR). Histopaque-1077, gelatin-free BSK H medium, dextran sulfate, and all other biochemicals were from Sigma (St. Louis, MO).

Analysis of Collagen-Positive Lymphocyte-like Cells of Rhesus Monkey Mononuclear Cells by Flow Cytometry

Monkey mononuclear cells were isolated from normal male Rhesus monkey blood on Histopaque-1077 according to the manufacturer's instructions. The cells were washed with phosphate-buffered saline (PBS) and incubated for 10 min at $37^{\circ} \mathrm{C}$ in PBS, $0.1 \% \mathrm{NaN}_{3}$ containing rabbit anti-human collagen IgG as the primary antibody. The cells then were washed in PBS-azide and incubated for $30 \mathrm{~min}$ at $37^{\circ} \mathrm{C}$ with FITCconjugated sheep anti-rabbit IgG. The cells were fixed with $1 \%$ paraformaldehyde and resolved on an EPICS 541 flow cytometer. In all experiments at least 10,000 cells were analyzed.

\section{In Vitro Cultivation of Spirochetes}

Low-passage $(<10)$ B. burgdorferi strain JDl was obtained from the Centers of Disease Control (CDC). B. burgdorferi M297 was obtained from Dr. Russell Johnson (University of Minnesota). The spirochetes were cultured according to Barbour (20) at $34^{\circ} \mathrm{C}$ (in $5 \% \mathrm{CO}_{2}, 3 \% \mathrm{O}_{2}$, and $92 \%$ $\mathrm{N}_{2}$ ) in gelatin-free BSK-H medium containing $10 \%$ young-rabbit serum. The bacteria were examined with a dark-field microscope to verify that the organisms were thoroughly dispersed at the start of all assays.

In Vitro Cultivation of Human and Rhesus Monkey Peripheral Blood Fibrocytes

Fibrocytes were harvested and cultured from human or rhesus monkey blood peripheral blood 

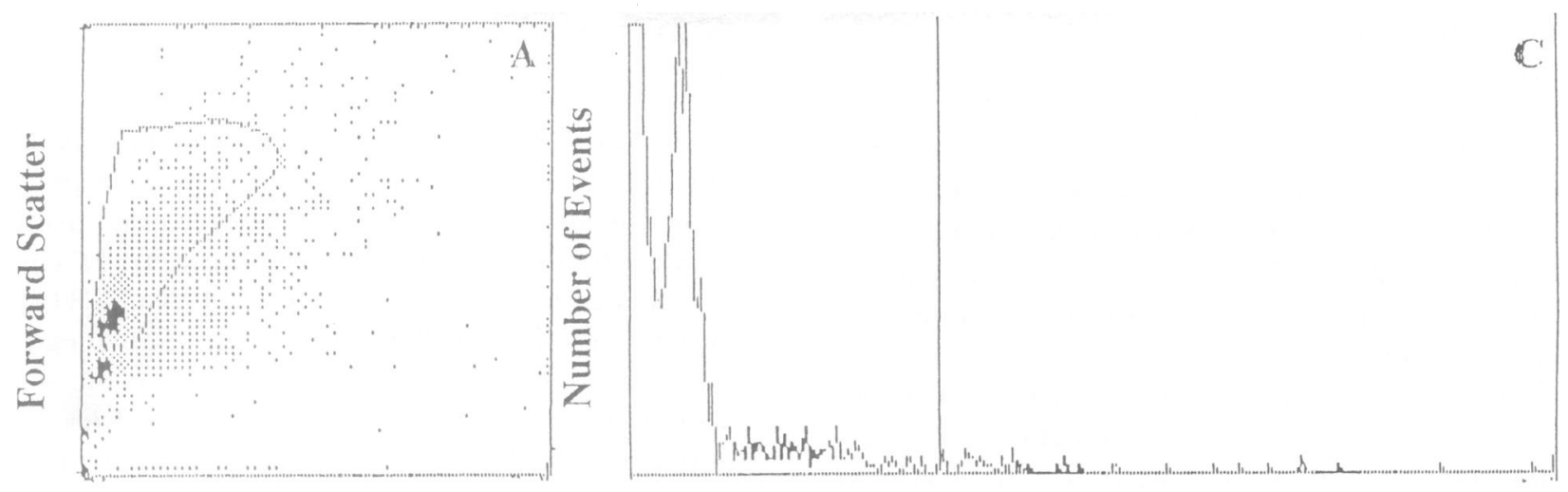

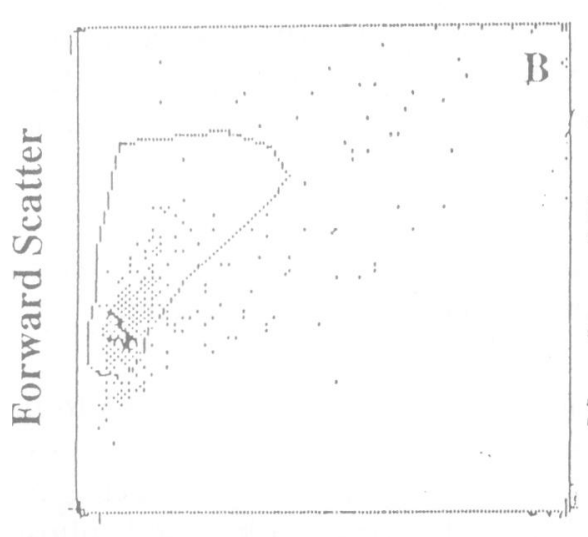

Sicle Scatter

Fig. 1. Flow cytometry analysis of collagenpositive lymphocyte-like cells of rhesus monkey peripheral blood mononuclear cells by flow cytometry. (A) Lymphocyte and monocyte region of light scatter plot of the stained cell preparation selected for analysis. (B) Brightly stained cells shown in (D) exhibited light scatter properties of

mononuclear cell preparations as previously described (15). Following 10 days of continuous culture, most of the attached human or monkey cells become morphologically transformed into elongated fibroblast-shaped cells. In addition to morphology, the purity of the fibrocyte (human) cultures was verified by flow cytometry analysis as previously described (15) using both phycoerythrin-conjugated anti-CD34 and FITC-conjugated anti-collagen type I MAb.

Interaction of B. burgdorferi with Peripheral Blood Fibrocytes and Mononuclear Cells

B. burgdorferi (approximately $10^{7}$ to $10^{8}$ cells $/ \mathrm{ml}$ in BSK-H medium), were directly added to a human peripheral blood mononuclear cell preparation or human or monkey fibrocyte cultures, usually at a $\mathrm{l}: 2$ ratio $(\mathrm{v}: \mathrm{v})$, and the cells were co-cultured at $34^{\circ} \mathrm{C}$ (in $5 \% \mathrm{CO}_{2}, 3 \% \mathrm{O}_{2}$, and $92 \% \mathrm{~N}_{2}$ ). B. burgdorferi binding to fibrocytes was monitored by dark-phase contrast microscopy using a Zeiss Axiovert 100 inverted light microscope.

For electron microscopy, the cells were fixed in situ with $2 \%(\mathrm{v} / \mathrm{v})$ glutaraldehyde in 0.1 M Na-cacodylate- $\mathrm{HCl}$ buffer at $\mathrm{pH}$ 7.3. After fixation, the cells were removed from the $\mathrm{T}$ flasks with a cell scraper, and further processing was done with $2 \%(\mathrm{w} / \mathrm{v}) \mathrm{OsO}_{4}$ and $0.5 \%$ (w/v) uranyl acetate. After ethanol dehydration, the cells were embedded in EMBed 812 via propylene-oxide. Thin sections were examined with a JEOL 1200EX II electron microscope. 

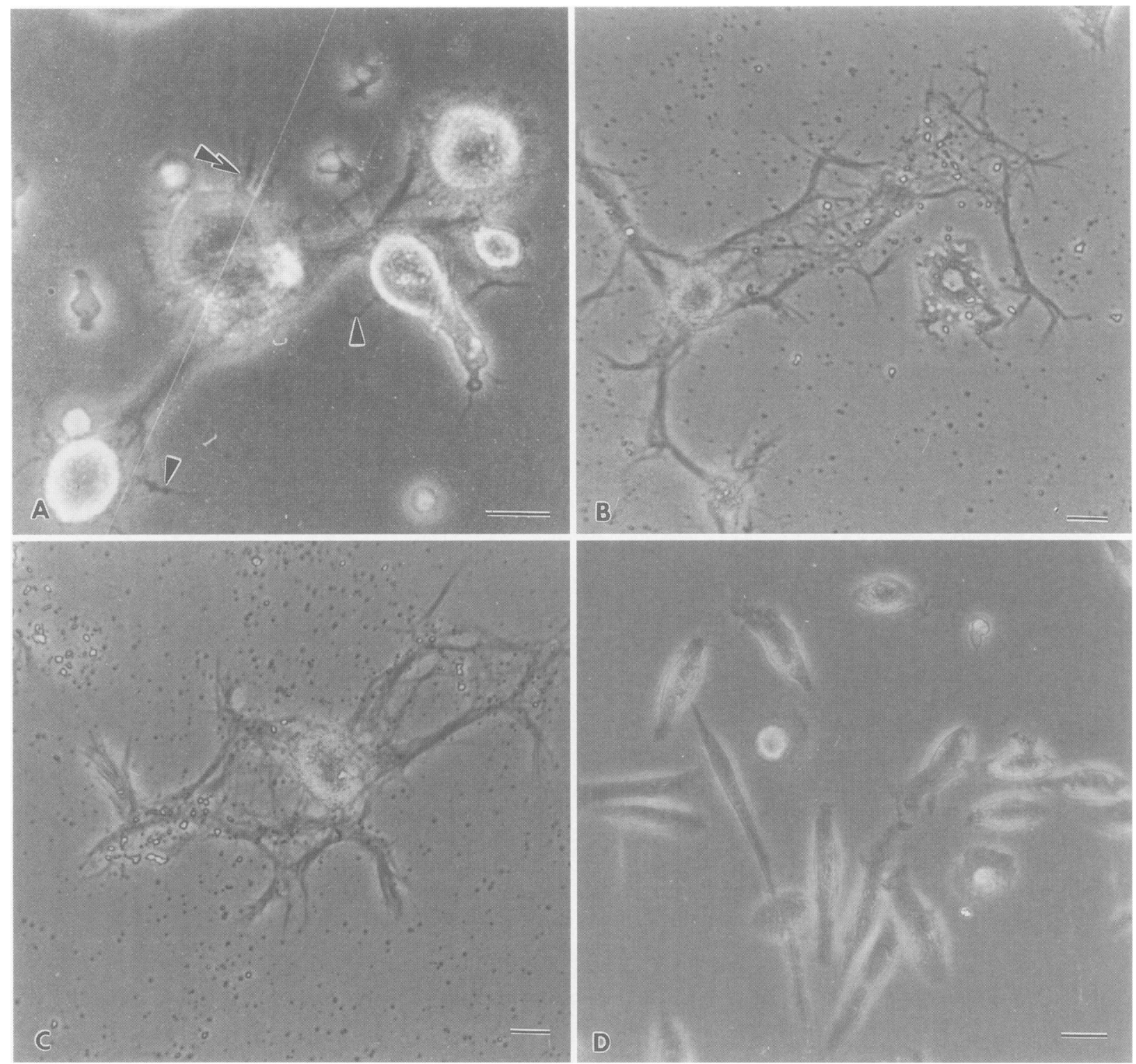

Fig. 2. Binding of B. burgdorferi to human and monkey peripheral blood fibrocytes. The binding of $B$. burgdorferi JDl to human fibrocytes after $30 \mathrm{~min}$ (A) or monkey fibrocytes after $30 \mathrm{~min}$ (B) or $45 \mathrm{~min}$ (C) coculture is shown. Control human fibrocytes are also shown (D). Incubation with M297 gave essentially similar results. In (A), single (arrowhead) and groups of (double arrowhead) spirochetes showed binding. (Bars $=10 \mu \mathrm{m}$ ).

\section{Results and Discussion}

The rhesus monkey is an important animal model for human Lyme disease $(21,22)$. Accordingly, we first purified and characterized circulating fibrocytes from the rhesus monkey. Rhesus monkey mononuclear cells were isolated and analyzed for fibrocyte content by flow cytometry (Fig. 1). The lymphocyte and monocyte region of the light scatter plot was selected for fibrocyte analysis using anti-collagen IgG. On the basis of experimental data obtained from 4 animals ( 1 female and 3 males), $6.1 \pm 3.3 \%$ (mean \pm standard deviation) of the cells with light scatter properties of lymphocytes stained positively for collagen. As in the human, where fibrocytes have been estimated to account for up to $0.5 \%$ of circulating leukocytes $(23,24)$, peripheral blood fibrocytes constitute an appreciable circulating cell population in the rhesus monkey.

We tested the ability of $B$. burgdorferi to interact with human (Fig. 2A) and with monkey peripheral blood fibrocytes (Fig. 2B, C). From 

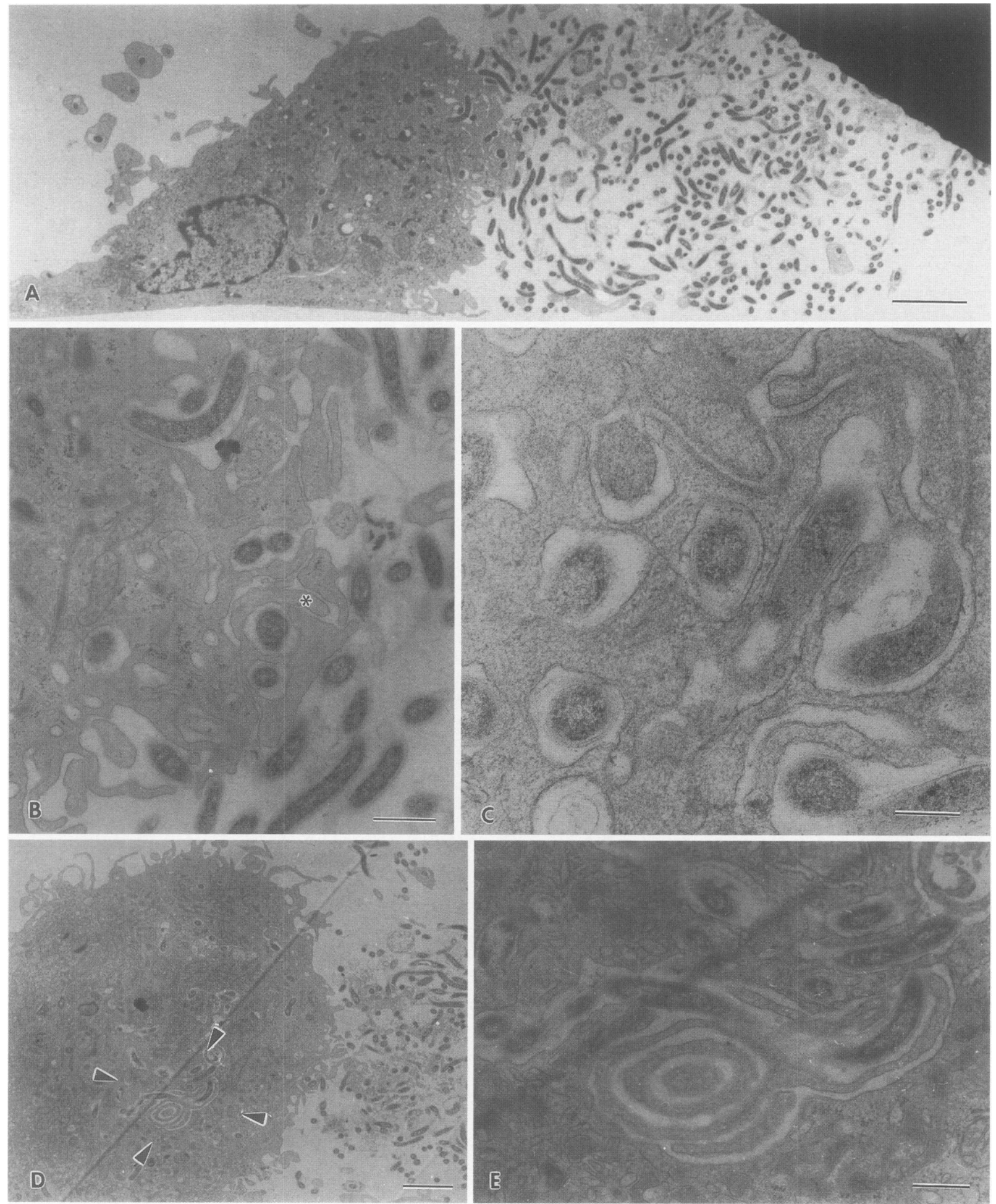

Fig. 3. Interaction of $B$. burgdorferi with peripheral blood fibrocytes. The figures represent fibrocyte/B. burgdorferi interactions of approximately 1-2 hr. (A) Fibrocyte sectioned perpendicular to substrate and showing polarized binding of spirochetes (M297). Bar $=4 \mu \mathrm{m}$. (B) Periphery of fibrocyte showing uptake of spirochetes. Some of the pseudopodial profiles resemble coiled phagocytosis (aster- isk). Bar $=500 \mathrm{~nm} .(\mathrm{C})$ Location of $B$. burgdorferi within recesses of the cell membrane, deep within the cytoplasm of a fibrocyte. Bar $=200 \mathrm{~nm}$. (D) Rounded fibrocyte. The arrowheads delineate the area enlarged in (E). Bar $=2 \mu \mathrm{m}$. (E) Enlargement from (D), delineated area. Spirochetes are located in deep invaginations of the cell membrane, deep within the cytoplasm. Bar $=500 \mathrm{~nm}$. 


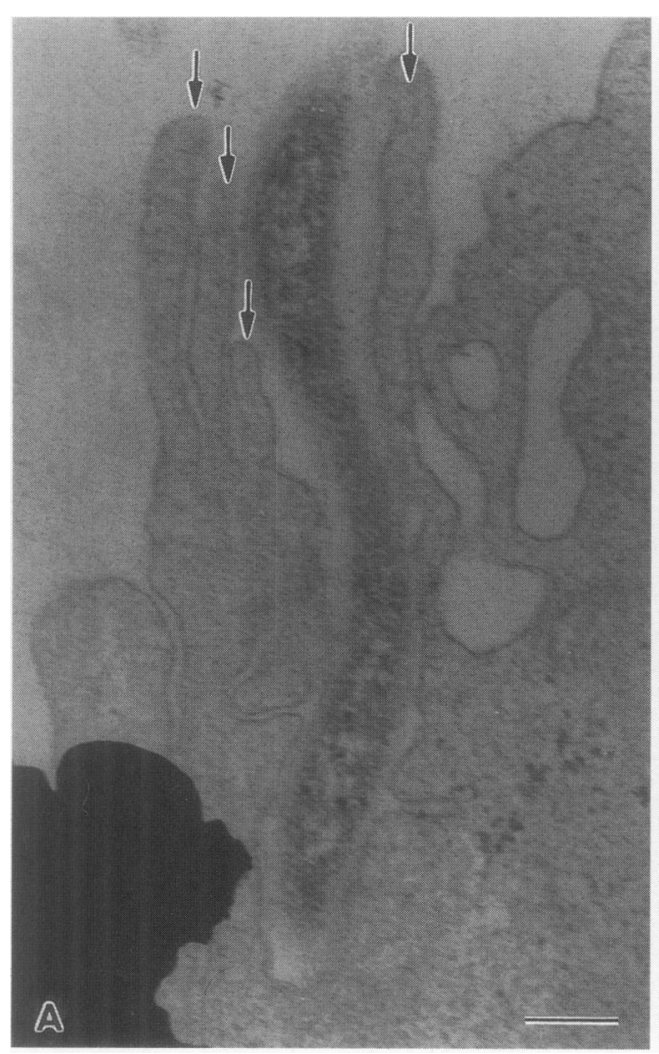

Fig. 4. Tube phagocytosis of $B$. burgdorferi by fibrocytes. (A) Longitudinal section through a spirochete cell being taken up by a fibrocyte. During uptake, the spirochete cell is surrounded by straight cell extensions (arrows). The appearance of these finger-like extensions is due to the section plane. These pseudopodial cell extensions actually form

light-microscopic observations it appears that $B$. burgdorferi spirochetes are able to bind via their tips to the surface of fibrocytes. The binding of single spirochetes (single arrowhead) or groups of spirochetes (double arrowhead) is marked in Figure 2A. Human fibrocyte controls are shown in Figure 2D. Borrelia interactions were the same whether we used $B$. burgdorferi JDl, a strain highly pathogenic in monkeys $(21,22)$, or $B$. burgdorferi M297, a strain lacking OspA and OspB (25). This implies that OspA and OspB do not play a role in Borrelia/fibrocyte interactions. From electron microscopic observations it appears that the binding of $B$. burgdorferi to fibrocytes is polarized to one side of the cell (Fig. 3A).

$B$. burgdorferi invades B cells through endocytotic pits into vacuoles (26). However, the entry of $B$. burgdorferi into B lymphocytes differs markedly from the phenomenon we observed for the interaction of spirochetes with peripheral blood fibrocytes. It appears that this uptake in-



cuffs that surround the spirochete. $\mathrm{Bar}=200 \mathrm{~nm}$. (B) Phagocytic tube in longitudinal section. A $B$. burgdorferi cell is sectioned in several places. The asterisk marks a phagocytic tube in cross section. (Insert) $B$. burgdorferi surrounded by two concentric phagocytic tubes in cross section. Bars ( $B$ and insert) $=500 \mathrm{~nm}$.

volves mechanisms that are different from those in conventional phagocytosis or coiling phagocytosis. The active uptake process known as "coiling" phagocytosis used by human phagocytic cells (i.e. monocytes, macrophages, polymorphonuclear leukocytes, dendritic cells, and synovial macrophages) for B. burgdorferi and other spirochetes has been documented recently (27-31). From the work by Rittig and co-workers it is clear that morphologically similar spirochetes can induce different frequencies of coiling phagocytosis (31). The frequency of coiling phagocytosis of different viable or killed highand low-passage strains of $B$. burgdorferi sensu strictu, B. garinii and $B$. afzelii, were reported to be within the same range of $40-60 \%$. Different strains of Treponema and Leptospira as well as relapsing fever Borrelia displayed a much lower frequency of coiling phagocytosis (from $30 \%$ to $<1 \%$ ).

As a first visible response to $B$. burgdorferi, we 


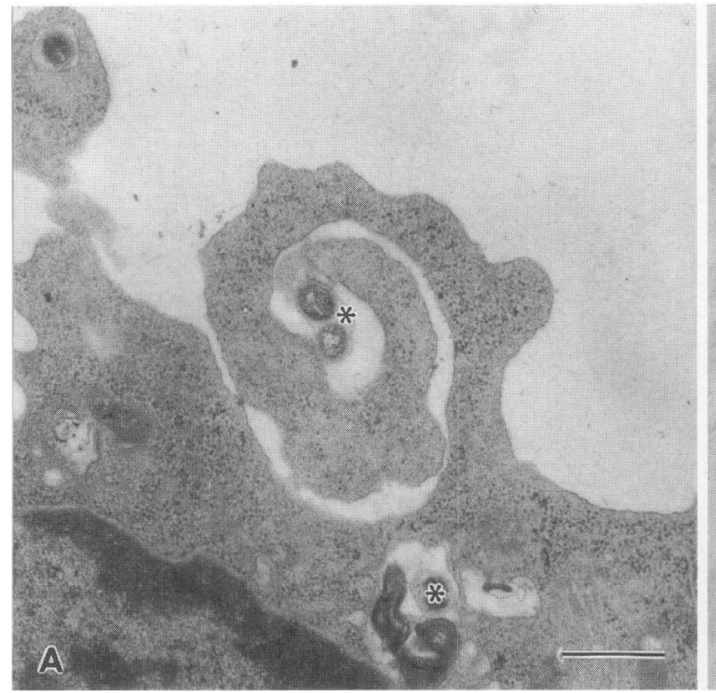

Fig. 5. Interaction of B. burgdorferi with human peripheral blood mononuclear cells. $\mathrm{Hu}$ man polymorphonuclear leukocytes and isolated peripheral blood fibrocytes were incubated with $B$. burgdorferi as described in Figures 2 and 3. (A) Coiling phagocytosis of peripheral blood mononuclear

observed that normally elongated or spindleshaped fibrocytes (Fig. 2D) become round (Figs. 2A-C, 3A). This morphologic change may occur for the fibrocyte to recruit sufficient plasma membrane to effect spirochete uptake. Indeed, it has been shown by scanning electron microscopy that peripheral blood fibrocytes display from the cell surface projections that are intermediate in size between pseudopodia and microvilli (15) (see also Fig. 3B). We believe that this is a specific response to the presence of spirochetes for the following reasons. The change in fibrocyte cell shape was not induced by incubating the cells at $34^{\circ} \mathrm{C}$ in the tri-gas mixture favored by the spirochetes, nor by addition of BSK-H medium. The binding itself is polarized to one side of the cell (Fig. 3A). Whether this is an induced receptor capping phenomenon or it has another molecular basis remains to be investigated.

Once binding has occurred, the spirochetes are not internalized into the fibrocyte cytoplasm but are first surrounded by slender, pseudopod-like extensions of the cell (Figs. 3B and 4A). Recently, the term "tube phagocytosis" was introduced to describe how B. burgdorferi invade neutrophils (32). We also see 'tubelike' structures extruding from fibrocytes and containing B. burgdorferi cells (Fig. 4B). These processes are formed on that side of the cell cells incubated with $B$. burgdorferi JDl for $2 \mathrm{hr}$. Two spirochetes (asterisks) are enveloped by a coiling pseudopodial cell extension. $\mathrm{Bar}=500 \mathrm{~nm}$. (B) Mononuclear cell with large phagocytic vacuoles (asterisks) containing spirochetes. Bar $=2 \mu \mathrm{m}$.

where spirochetes are bound (cf. Fig. 3A, the right side of the cell with the spirochete-free left side of the pictured cell). We conclude that these structures are a cellular response to $B$. burgdorferi spirochetes, perhaps as a consequence of receptor capping. The Borelliae are then enveloped into recesses of the cell membrane projecting deep into the cytoplasm (Fig. 3C, D, E). These recesses actually form an anastomosing coiled and twisted network within the fibrocyte with $B$. burgdorferi cells interspersed (Fig. 3C, E). Occasionally we found profiles that resemble to some degree classical coiling phagocytosis (Fig. 3B, asterisk), but these are probably accidental configurations. Further studies using other $B$. burgdorferi strains will determine the universality of spirochete entry into fibrocytes.

When incubated with a human peripheral blood mononuclear cell preparation, we find many profiles of phagocytic cells where the Borreliae are internalized by coiling phagocytosis, as illustrated in Figure 5A. Following this process, they are taken up into large intracellular vacuoles (Fig. 5B). This confirms the significant findings by Rittig and co-workers $(28-31)$ and illustrates the difference between $B$. burgdorferi uptake into leukocytes and into fibrocytes.

In conclusion, we found that $B$. burgdorferi binds to fibrocytes and resides within deep in- 
vaginations on the cell surface. It has been reported that fibrocytes display prominent cell surface projections, intermediate in size between microvilli and pseudopodia (15). Because of the spirochetes' corkscrew movement, it is possible that the spirochetes wrap themselves up within these membrane projections without actually being endocytosed. This kind of "internalization" within the peripheral blood fibrocyte may protect $B$. burgdorferi not only from the host immune system but also from the fibrocyte's lysosomaldigestive system. It recently has been shown that B. burgdorferi envelope themselves with layers of lymphocyte membrane as they exit some spirochete-infected lymphocytes and that this membrane-cloaking mechanism may protect the spirochete from humoral and cellular recognition (26). The process by which $B$. burgdorferi can exit the peripheral blood fibrocyte remains to be determined. We hypothesize that the fibrocytes carry the spirochetes to the connective tissues. With respect to the tick vector, it is possible that spirochete-infected fibrocytes have the ability to migrate to the site of tick attachment and infect the arthropod vector. The observation that under the influence of certain physiological signals, fibrocytes also possess the functional capacity to recruit, activate, and present antigen to $\mathrm{CD}^{+}{ }^{+} \mathrm{T}$ cells (23) suggests that perhaps other modes of spirochete entry into these cells are possible. Finally, our findings, in combination with these recent discoveries, suggest that peripheral blood fibrocytes have the potential to play an important role in the immunopathology of Lyme arthritis.

\section{Acknowledgments}

We especially acknowledge the expert technical help provided by Calvin Lancloe, Richard Kennedy, and Christina Givens (Tulane Regional Primate Research Center). We are also thankful to both Dr. Mark Wiser (Tulane University School of Public Health and Tropical Medicine) and Dr. Peter Didier (Tulane Regional Primate Research Center) for their most helpful discussions. This work was presented in part at the Experimental Biology '97 Meetings held in New Orleans, LA, April 6-9, 1997 (33). Dennis J. Grab and H.-Norbert Lanners contributed equally to this work. Financial support for this work was provided in part by grants from the CDC (U50/CCU606604-05) and from the NCRR (P5IRRAG00164-35).

\section{References}

1. Steere AC. (1989) Lyme disease. N. Engl. J. Med. 321: $586-596$.

2. Centers for Disease Control. (1992) Notifiable diseases report. Morbid. Mortal. Weekly Rep. 40: 898899.

3. Coleman JL, Sellati TJ, Testa JE, et al. (1995) Borrelia burgdorferi binds plasminogen, resulting in enhanced penetration of endothelial monolayers. Infect. Immun. 63: 2478-2484.

4. Fuchs H, Wallich R, Simon MM, Kramer MD. (1994) The outer surface protein A of the spirochete Borrelia burgdorferi is a plasmin(ogen) receptor. Proc. Natl. Acad. Sci. U.S.A. 91: 12594-12598.

5. Hu LT, Perides G, Noring R, Klempner MS. (1995) Binding of human plasminogen to Borrelia burgdorferi. Infect. Immun. 63: 3491-3496.

6. Klempner MS, Noring R, Epstein MP, et al. (1994) Subversion of the host fibrinolytic pathway for invasion by the Lyme disease spirochete, Borrelia burgdorferi. In: Cevenini R, Sambri V, La Placc M (eds.) Advances in Lyme Research. Proceedings of the VI International Conference on Lyme borreliosis. pp. 997-100.

7. Klempner MS, Noring R, Epstein MP, et al. (1995) Binding of human plasminogen and urokinasetype plasminogen activator to the Lyme disease spirochete, Borrelia burgdorferi. J. Infect. Dis. 171: 1258-1265.

8. Grab DJ, Kennedy R, Philipp MT. (1996) Borrelia burgdorferi possess a collagenolytic activity. FEMS Microbiol. Lett. 144: 39-45.

9. Grab DJ, Didier P, Lanners HN, Adkisson HD. (1996) Proteoglycan degradation and induction of nitric oxide synthase activity of human articular cartilage by Borrelia burgdorferi. Presented at IBC 6th Annual Conference on New Therapeutic Advances in Arthritis, December 9-10, New Orleans, LA.

10. Williams LR, Austin FE. (1992) Hemolytic activity of Borrelia burgdorferi. Infect. Immun. 60: 32243230.

11. Georgilis K, Peacocke M, Klempner MS. (1992) Fibroblasts protect the Lyme disease spirochete, Borrelia burgdorferi, from ceftrixone in vitro. J. Infect. Dis. 166: 440-444.

12. Klempner MS, Noring R, Rogers RA. (1993) Invasion of human skin fibroblasts by the Lyme disease spirochete, Borrelia burgdorferi. J. Infect. Dis. 167: 1074-1081.

13. Pachner AR, Basta J, Delaney E, Hulinska D. (1995) Localization of Borrelia burgdorferi in murine Lyme borreliosis by electron microscopy. Am. J. Trop. Med. Hyg. 52: 128-123.

14. Coburn J, Leong JM, Erban JK. (1993) Integrin $\alpha_{\mathrm{IIb}} \beta_{3}$ mediates binding of the Lyme disease agent Borrelia burgdorferi to human platelets. Proc. Natl. Acad. Sci. U.S.A. 90: 7059-7063.

15. Bucala R, Spiegel LA, Chesney J, et al. (1994) 
Circulating fibrocytes define a new leukocyte subpopulation that mediates tissue repair. Mol. Med. 1: 71-81.

16. Szczepanski A, Furie MB, Benach JL, et al. (1990) Interaction between Borrelia burgdorferi and endothelium cells in vitro. J. Clin. Invest. 85: 16371647.

17. Grab DJ, Givens C, Kennedy R. (1998) Fibronectin-binding activity in Borrelia burgdorferi. Biochim. Biophys. Acta 1407: 135-145.

18. Barthold SW, Persing $\mathrm{DH}$, Armstrong $\mathrm{AL}$, et al. (1991) Kinetics of Borrelia burgdorferi dissemination and evolution of disease after intradermal inoculation of mice. Am. J. Pathol. 139: 263-273.

19. Häupl T, Hahn G, Rittig M, et al. (1993) Persistence of Borrelia burgdorferi in ligamentous tissue from a patient with chronic Lyme borreliosis. $A r$ thritis Rheum. 36: 1621-1626.

20. Barbour AG. (1984) Isolation and cultivation of Lyme disease spirochetes. Yale J. Biol. Med. 57: 521-525.

21. Philipp MT, Aydintug MK, Bohm RP Jr, et al. (1993) Early and early disseminated phases of Lyme disease in the rhesus monkey: a model for infection in humans. Infect. Immun. 61: 30473059.

22. Roberts D, Bohm RP Jr, Cogswell FB, et al. (1995) Chronic Lyme disease in the rhesus monkey. Lab. Invest. 72: 146-160.

23. Chesney J, Bacher M, Bender A, et al. (1997) The peripheral blood fibrocyte is a potent antigen-presenting cell capable of priming naive $\mathrm{T}$ cells in situ. Proc. Natl. Acad. Sci. U.S.A. 94: 6307-6312.

24. Chesney J, Metz C, Stavitsky AB, et al. (1998) Regulated production of type I collagen and inflamatory cytokines by peripheral blood fibrocytes. J. Immunol. 160: 419-425.

25. Hughes CA, Engstrom SM, Coleman LA, et al. (1993) Protective immunity is induced by a Bor- relia burgdorferi mutant that lacks OspA and OspB. Infect. Immun. 61: 5115-5122.

26. Dorward DW, Fischer ER. (1997) Lymphocyte invasion and host-cell membrane cloaking protects Borrelia burgdorferi from complement-mediated killing. In: 97th General Meeting of the American Society for Microbiology (May 4-8, Miami Beach, FL) Abstract D-133, ASM Press, Washington, D.C.

27. Montgomery RR, Malawista SE. (1996) Entry of Borrelia burgdorferi into macrophages is end-on and leads to degradation in lyosomes. Infect. Immun. 64: 2867-2872.

28. Rittig MG, Häupl T, Krause A, et al. (1994) Borrelia burgdorferi-induced ultrastructural alterations in human phagocytes: a clue to pathogenicity. J. Pathol. 173: 269-282.

29. Filgueira L, Nestle FO, Rittig M, Joller HI, Groscuth P. (1996) Human dendritic cells phagocytose and process Borrelia burgdorferi. J. Immunol. 7: 2998-3005.

30. Rittig MG, Kuhn KH, Dechant CA, et al. (1996) Phagocytes from both vertebrate and invertebrate species use "coiling" phagocytosis. Dev. Comp. Immunol. 20: 393-406.

31. Rittig MG, Jagoda JC, Wilske B, et al. (1998) Coiling phagocytosis discriminates between different spirochetes and is enhanced by phorbol myristate acetate and granulocyte-macrophage colony-stimulating factor. Infect. Immun. 66: 627635.

32. Suhonen J, Hartiala K, Viljanen MK. (1998) Tube Phagocytosis, a novel way for neutrophils to phagocytize Borrelia burgdorferi. Infect. Immun. 66: 3433-3435.

33. Grab DJ, Lanners N, Didier P, Chesney J, Givens C, Kennedy R, Bucala R. (1997) Borrelia burgdorferi bind to peripheral blood fibrocytes: leukocytelike cells with potential for connective tissue targeting. FASEB J. 11: Al15. 